\title{
Inseguridad Laboral Percibida y Malestar Psicológico: Evaluación de Dos Modelos Predictivos en Trabajadores Suministrados de la Ciudad de Concepción, Chile
}

\section{PERCEIVED JOB INSECURITY AND PSYCHOLOGICAL DISCOMFORT: EVALUATION OF TWO PREDICTIVE MODELS IN SUPPLIED WORKERS OF CONCEPCIÓN CITY, CHILE}

Leonardo Valencia Silva', Cecilia Avendaño Bravo²

1. Psicólogo. Magister en Psicología de la Salud. Egresado postgrado de la Facultad de Ciencias Sociales, Universidad de Concepción, Chile.

2. Psicóloga. Doctora en Psicología. Profesora de la Facultad de Ciencias Sociales, Universidad de Concepción, Chile.

\section{RESUMEN}

El objetivo del estudio fue evaluar dos modelos teóricos sobre la relación entre inseguridad laboral percibida y malestar psicológico, y sus variables predictoras (sexo, edad, situación de pareja, escolaridad y tipo de contrato), en 112 trabajadores suministrados de Concepción. El estudio es transversal de tipo explicativo. La recolección de los datos fue realizada mediante un muestreo intencionado. Los instrumentos utilizados fueron el Cuestionario de Salud General de Goldberg y Williams ${ }^{1}$, una escala de inseguridad laboral percibida cuantitativa y otra cualitativa ${ }^{2}$ y una encuesta con datos sociodemográficos-laborales. El análisis de las relaciones causales se realizó con el método de Análisis de Ecuación Estructural (SEM). Los resultados indican que los modelos no son aplicables a trabajadores suministrados chilenos. Ello podría deberse a diferencias en las situaciones de trabajo y formas de flexibilidad laboral de los participantes, en las condiciones estructurantes del empleo suministrado en Chile, y a la influencia de variables individuales asociadas con la subjetividad de los trabajadores.

(Valencia L, Avendaño C, 2014. Inseguridad Laboral Percibida y Malestar Psicológico: Evaluación de Dos Modelos Predictivos en Trabajadores Suministrados de la Ciudad de Concepción, Chile. Cienc Trab. Ene-Abr; 16 [49]: 49-55).

Palabras claves: INSEGURIDAD LABORAL PERCIBIDA; MALESTAR PSICOLÓGICO; TRABAJO SUMINISTRADO; ANÁLISIS DE ECUACIONES ESTRUCTURALES.

\section{ABSTRACT}

The objective of the study was evaluating two theorical models on relation between perceived job insecurity and psychological discomfort, and their predictive variables (sex, age, marital status, educational level and type of contract), in 112 supplied workers of Concepción. It is a cross sectional and explanatory study. The analysis of causal relationships was made using the method of Structural Equation Modeling (SEM). The results indicate that the models are not applicable on Chilean supplied workers. This could be due to differences in work situations and forms of labor flexibility of the participants, in the structural conditions of employment provided in Chile, and the influence of individual variables associated with the subjectivity of workers.

Key words: PERCEIVED JOB INSECURITY; PSYCHOLOGICAL DISTRESS; WORK PROVIDED; STRUCTURAL EQUATION ANALYSIS.

\section{INTRODUCCIÓN}

En las últimas décadas, el contexto de cambios profundos en el mundo del trabajo ha impulsado el desarrollo de diversas formas de reorganización de las empresas, mediante las cuales buscan aumentar su flexibilidad para adaptarse a las nuevas demandas

\section{Correspondencia / Correspondence:}

Leonardo Valencia Silva

Departamento de Psicología, Facultad de Ciencias Sociales,

Universidad de Concepción

Barrio Universitario $s / n$, Concepción, Chile

Tel.: 2203947

e-mail: e.leonardo.valencia@gmail.com

Recibido: 24 Enero 2014 / Aceptado: 13 de Febrero 2014

en escenarios altamente competitivos. Aunque diversas, estas formas implican frecuentemente acciones de tercerización, privatización y fusiones en combinación con tácticas de reducción de personal y un aumento de la subcontratación, observándose que en común tienden a elevar la preocupación de los trabajadores respecto a su futuro en general y al de su trabajo. ${ }^{3}$ En Chile, el trabajo suministrado se ha generalizado de modo progresivo, particularmente en la gran y mediana empresa ${ }^{4}$, incorporado entre enero del 2010 y octubre del 2013 a 295.566 empleados, 103.872 hombres y 191.694 mujeres $^{5}$, mientras que para el año 2011 la encuesta ENCLA identificaba sólo 98.855. ${ }^{6}$

En el trabajo suministrado (TS), los trabajadores son contratados por una agencia de empleo temporal (EST) que los envía a desempeñarse en diferentes organizaciones clientes, generándose una relación laboral "triangular" entre trabajadores, organización usuaria y agencia suministradora. Así, el núcleo del TS está conformado por relaciones múltiples, enlaces débiles entre orga- 
nización cliente y trabajadores, y transiciones en la trayectoria de cada trabajador entre las diferentes organizaciones asignadas. ${ }^{7}$ Cada asignación a una organización distinta implica un nuevo lugar de trabajo, con diferentes deberes y funciones, otros compañeros de trabajo y supervisores locales, y otra cultura organizacional $^{8}$, viéndose enfrentados a asimilar los procedimientos de la nueva organización, a menudo con falta de apoyo de compañeros y supervisores de trabajo. ${ }^{9}$ Además, los trabajadores suministrados poseen mayoritariamente bajas calificaciones para las tareas en la actividad principal de la empresa cliente, son destinados principalmente a trabajos de baja complejidad y alta inseguridad, reciben salarios más bajos aun cuando ejecuten tareas de trabajo similares a quienes tienen puestos de trabajo permanentes, y tienen menos control sobre el contenido de su trabajo y la duración de la jornada. ${ }^{10}$ La razón principal de la mayoría de los trabajadores para tomar un TS sería la falta de alternativas de empleo, prefiriendo un empleo estable y esperando una oferta para convertirse en empleado permanente de la organización usuaria. $^{7}$

Aunque diversos estudios han encontrado factores de riesgo semejantes para el trabajo temporal y otros tipos de empleo, y algunas características del trabajo temporal -como la presión económica- son compartidas con otros trabajadores en situación precaria, investigaciones recientes muestran que algunos riesgos son exclusivos del contexto de TS e implican mayor riesgo de daño a la salud en los trabajadores suministrados o que estos sean más agudos que para otros trabajadores temporales. ${ }^{11}$ Muchos de estos riesgos estarían asociados con la relación laboral triangular del TS y su falta de regulación, y a que ni la EST ni la organización cliente aceptan la responsabilidad por la salud y seguridad laboral de los trabajadores. Underhill y Quinlan ${ }^{11}$ identifican riesgos de desorganización que se extienden a colocaciones inadecuadas, falta de familiaridad con los lugares de trabajo asignados y a una fractura de la comunicación.

\section{Inseguridad Laboral Percibida y Malestar Psicológico}

A mediados de los 80, la investigación en Psicología comenzó a concebir la inseguridad laboral (IL) como un factor de estrés. ${ }^{12,13}$ En general los investigadores han definido y operacionalizado la IL desde una perspectiva global que destaca las percepciones de amenaza de la pérdida del empleo o de la existencia del empleo ${ }^{12}$, pero otros la conceptualizan en términos multidimensionales señalando que no proviene solamente de la amenaza del desempleo sino también de la pérdida de ciertas dimensiones valoradas del empleo como la pérdida de oportunidades de promoción, aumento de sueldo $\mathrm{u}$ otras características importantes. ${ }^{14}$ En particular, Sverke et $\mathrm{al}^{3}$ asumen una distinción formulada por Hellgren et al. ${ }^{15}$ (1999) de dos aspectos de la inseguridad laboral: cuantitativo (preocupaciones referidas a la pérdida del trabajo) y cualitativo (preocupaciones sobre la pérdida de características valoradas del trabajo), similares a las perspectivas recién señaladas, argumentando que podrían ser significativas en la medición pero que aún sería necesario más desarrollo conceptual y empírico para aclarar los aspectos que en definitiva debieran incluirse en los estudios.

Todas las perspectivas anteriores comparten la idea que la IL es un fenómeno perceptual subjetivo caracterizado por su carácter anticipatorio ante un suceso importante de naturaleza involuntaria. ${ }^{3,12,13,16}$ Las percepciones de los individuos son vistas como el vínculo entre la situación laboral objetiva y las consecuencias negativas asociadas, y la IL surgiría en la interacción entre carac- terísticas contextuales y características del individuo que influyen en su interpretación de los factores del ambiente, lo que permitiría entender las diferencias encontradas entre trabajadores. ${ }^{3,16}$

El hecho que la percepción de IL vaya asociada con reacciones negativas sería predecible tanto porque sus componentes principales son el alto nivel de incertidumbre y la falta de control de las condiciones de trabajo como porque el trabajo cubre necesidades económicas y psicosociales importantes. ${ }^{17} \mathrm{La}$ evidencia muestra que está asociada con reacciones adversas e incluso la posiciona como el factor predictivo más importante de reacciones psicológicas tales como ansiedad, depresión y problemas psicosomáticos en los trabajadores ${ }^{3,18-20}$, de más sentimientos negativos, malestar y agotamiento en comparación con quienes experimentan seguridad en su empleo ${ }^{18}$, y de una peor autopercepción de salud general. ${ }^{17,21-23}$ Además, las percepciones de IL tendrían una influencia autónoma y perjudicial para la salud mental ${ }^{24}$, y cuando se controlan los efectos de otras condiciones estresantes del trabajo, la incertidumbre ante la pérdida del empleo tiene un efecto independiente. ${ }^{25}$

En conjunto, los hallazgos apoyan la noción que el trabajo en situación de inseguridad tiene efectos psicológicos perjudiciales para las personas y puede traer asociados importantes costos para las organizaciones, pues conduce a la enfermedad. ${ }^{26}$ La IL resulta disfuncional para el individuo porque afecta su salud, pero también para la organización, puesto que al influir sobre sus actitudes y comportamientos acaba repercutiendo sobre la eficacia; en el nivel individual produce falta de satisfacción y de implicación laboral a corto plazo, y aumenta la propensión al abandono y disminuye el rendimiento a largo plazo. ${ }^{12,27}$

Una revisión teórica de Sverke et $\mathrm{al}^{3}$, que incluye los resultados de un meta-análisis de Sverke et $\mathrm{al}^{12}$, propone los siguientes factores sociodemográficos y laborales como influyentes en la vulnerabilidad de los trabajadores a la IL percibida: (a) Edad. Los trabajadores entre 30 y 40 años responsables de la crianza de hijos experimentan la posibilidad de pérdida de empleo más negativamente que aquellos responsables sólo de su propio sustento, y los trabajadores mayores experimentan más inseguridad porque puede resultarles más difícil conseguir un nuevo empleo. (b) Género. Los hombres informan más inseguridad laboral que las mujeres debido a que tradicionalmente tienen asignado el rol de sostén económico de la familia y su identidad estaría más amenazada frente a la posible pérdida del empleo. (c) Estatus socioeconómico. Las personas con bajos ingresos y menor nivel de calificación son más vulnerables a la amenaza de la pérdida del empleo porque son más dependientes de la mantención de su empleo. (d) Tipo de contrato. Quienes tienen contrato indefinido o de tiempo completo informan niveles de inseguridad laboral menores que los trabajadores temporales o de tiempo parcial, lo cual se explicaría por un sentimiento más fuerte de "ser parte" de la organización.

Los resultados de un meta-análisis posterior ${ }^{28}$ replican los hallazgos anteriores sobre el efecto de la IL en la salud para estos factores, pero encontraron que el género no modera las relaciones entre IL y sus consecuencias, atribuyéndolo a que las mujeres están más comprometidas con su trabajo, tienen una movilidad ocupacional comparable con la de los hombres y ocupan a menudo el rol de jefas de hogar, y concluye destacando su impacto negativo y las diferentes maneras en que diversos tipos de empleados sufren y reaccionan.

Con base en los antecedentes expuestos, fue diseñado un estudio transversal que tuvo como objetivo probar en trabajadores suministrados chilenos dos modelos teóricos elaborados para el 
contexto laboral sueco sobre la relación entre ILP y malestar psicológico y sus variables predictoras (uno con ILP cuantitativa y otro con ILP cualitativa), que fueron evaluadas en trabajadores con condiciones flexibles de empleo. Así, estudios realizados con el primer modelo confirmaron que la ILP cuantitativa predice el malestar psicológico. ${ }^{24}$ Por otra parte, la evidencia parcial proveniente de otros estudios realizados principalmente en Europa llevó a proponer un segundo modelo que incluye ILP cualitativa como predictora del malestar psicológico ${ }^{3}$; entre otros, Boya et $\mathrm{al}^{2}$ encontraron que tanto la ILP cuantitativa como cualitativa afectan el bienestar psicológico. Ambos modelos constituyen un avance teórico significativo en la investigación desarrollada en el área al especificar relaciones complejas entre variables antes dispersas, al mismo tiempo que permiten observar dos formas de medir ILP, cuestión metodológica aún pendiente. Si bien en Chile y otros países latinoamericanos no existen estudios previos que aborden específicamente la relación aquí abordada9 , a la luz del conocimiento existente se consideró que poner a prueba estos modelos en trabajadores chilenos con empleo flexible en la creciente modalidad de TS permitiría la formulación de hipótesis causales sustentadas empíricamente relevantes sobre esta problemática para nuestra realidad.

Para la prueba del primer modelo fueron formuladas las siguientes hipótesis: a) La ILP cuantitativa predice el malestar psicológico; y, b) Los antecedentes sociodemográficos (sexo, edad, situación de pareja y escolaridad) y el tipo de contrato predicen la ILP cuantitativa y el malestar psicológico. Y para el segundo modelo: a) La ILP cualitativa predice el malestar psicológico; y, b) Los antecedentes sociodemográficos (sexo, edad, situación de pareja y escolaridad) y el tipo de contrato predicen la ILP cualitativa y el malestar psicológico.

\section{MATERIAL Y MÉTODOS}

\section{Participantes}

Los participantes son 112 trabajadores de EST que desempeñan actividades que requieren un bajo nivel de calificación en el área de los servicios de promoción y ventas, contacto telefónico, aseo, manipulación de alimentos y seguridad en el sector servicios. Las empresas usuarias en que realizan su trabajo son grandes tiendas de retail, centros de llamados, universidades y hospitales. La muestra fue seleccionada en forma intencionada.

\section{Instrumentos}

Las variables de estudio e instrumentos utilizados para evaluarlas son los siguientes:

1. Inseguridad Laboral Percibida: Evaluada con un instrumento elaborado por Boya et $\mathrm{al}^{2}$ que consta de una escala que evalúa inseguridad laboral percibida cuantitativa y otra que evalúa inseguridad laboral percibida cualitativa. La inseguridad laboral cuantitativa es medida con 5 ítems basados en elementos desarrollados por Ashford et $\mathrm{al}^{29}$, Hellgren et $\mathrm{al}^{15}$ y De Witte ${ }^{30}$, y los aspectos cualitativos con 4 items basados en una escala de Isaksson et al. ${ }^{31}$ Los ítems están redactados como afirmaciones y tienen 5 alternativas de respuesta (desde 1 totalmente en desacuerdo hasta 5 totalmente de acuerdo). Una puntuación total elevada corresponde a un alto nivel de inseguridad en el empleo. La confiabilidad estimada con el coeficiente Alpha de Cronbach es 0,73 para la escala cuantitativa y 0,83 para la escala cualitativa. ${ }^{2}$ En el análisis de confiabilidad con los datos obtenidos en el presente estudio, se eliminó un ítem de la escala cuantitativa (ítem 2) y otro de la escala cualitativa (item 3) por su baja correlación con los demás elementos. Así, el coeficiente obtenido para la escala cuantitativa es 0,891 y para la escala cualitativa 0,803 .

2. Malestar psicológico: Se utilizó el Cuestionario de Salud General ${ }^{1}$ que evalúa la autopercepción del estado de salud mental, en su versión de 30 ítems. Es un cuestionario autoadministrable consistente en un listado de frases en que el respondiente compara un determinado aspecto de su experiencia reciente con su estado habitual. Las alternativas de respuesta son 4 y se puntúan de 0 a 4 puntos. La puntuación total es la suma de las puntuaciones para cada item, y una puntuación mayor es indicativa de una percepción de peor estado de salud mental. Esta versión ha sido utilizada en Chile desde los años 70 en investigaciones con trabajadores y su consistencia interna estimada con el coeficiente Alpha de Cronbach es $0,90 .^{32}$

3. Las variables sociodemográficas y laborales consideradas fueron: sexo ( 0 = hombre, 1 = mujer); edad (rangos 18-24, 25-31, 32-38, 39-45, 46-52, 53-59 y 60 años y más); situación de pareja ( 0 = sin pareja estable, 1 = con pareja estable); nivel de escolaridad $(0=$ bajo, $1=$ alto $)$, y tipo de contrato $(0=$ indefinido, $1=$ temporal). Para recabar esta información fue diseñada una encuesta específica.

En los modelos de ecuaciones estructurales, las variables latentes inseguridad laboral percibida cuantitativa y cualitativa fueron representadas por los items resultantes del análisis de confiabilidad del instrumento de Boya et $\mathrm{al}^{2}$ realizado en este estudio. La variable latente malestar psicológico fue representada por cuatro indicadores (ansiedad, depresión, alteraciones del sueño y síntomas físicos), de acuerdo con lo sugerido por Avendaño y Román ${ }^{32}$, con el propósito de reducir el número de variables observadas. Las variables sociodemográficas y tipo de contrato fueron incluidas en el análisis SEM como variables observadas.

\section{Procedimiento}

La recolección de datos se realizó entre abril y julio de 2012. Para contactar posibles participantes fue utilizada la técnica de bola de nieve con informantes claves. Previo a la aplicación, los participantes firmaron una carta de consentimiento informado que enfatiza la voluntariedad de su participación y la confidencialidad de sus datos personales, y la importancia de responder todos los ítems del instrumento. La aplicación fue en el lugar de trabajo del participante y duró aproximadamente 25 minutos. Este estudio recibió la aprobación de la Comisión Evaluadora del Programa de Magíster en Psicología, Universidad de Concepción.

En el análisis descriptivo se utilizaron medidas de tendencia central y dispersión, t de Student y Anova. En el análisis de las relaciones causales se aplicó el método de Análisis de Ecuación Estructural (SEM) del programa AMOS v.18, siguiendo las etapas de especificación, identificación, estimación de parámetros, evaluación de ajuste del modelo, modificación del modelo e interpretación de los resultados. ${ }^{33}$ En el análisis de los modelos se utilizaron los procedimientos de estimación de máxima verosimilitud, y la calidad se evaluó con los índices de bondad de ajuste sugeridos por Ruiz et al. ${ }^{34}$ Para la evaluación global del modelo estructural se utilizó la prueba de Chi-cuadrado y la Razón Chi-cuadrado/grados de libertad, considerando como criterio que si Chi-cuadrado tiene una significancia menor o igual a $0,05 \mathrm{el}$ modelo debe rechazarse por no reproducir los datos de la muestra observada. ${ }^{35}$ Además, fueron utilizados los siguientes índices 
complementarios: Raíz del residuo cuadrático promedio de aproximación (RMSEA) que debe tener un valor menor a 0,08; Índice de bondad de ajuste (GFI) e Índice de bondad de ajuste comparativo (CFI), que deben tener valores mayores a $0,95 .{ }^{33} \mathrm{Un}$ modelo tendrá un "buen ajuste" con los datos observados cuando gran parte de sus indices de ajuste cumplen con los criterios de aceptación establecidos y la totalidad de las relaciones que contiene son significativas. ${ }^{35}$

\section{RESULTADOS}

\section{Resultados descriptivos}

La muestra está compuesta por 55 hombres y 57 mujeres con una edad media de 34,5 años (DS = 11,6) en un rango de 18 a 64 años. La mayoría son jóvenes de 38 y menos años (69\%), con pareja estable (81\%), y niveles de escolaridad mediano y alto (36\% y $43 \%$ ). Un 61\% tiene contrato de duración indefinida y el 39\% restante mantiene alguna forma contractual de tipo temporal, fluctuando entre menos de un año y 13 años de trabajo en la misma EST. La proporción de participantes con contrato indefinido es superior en hombres (57\%), mientras que con contrato temporal es mayor la proporción de mujeres (64\%), diferencias estadísticamente significativas ( $\left.\_2=0,201, \mathrm{p}=0,03\right)$.

En la escala de inseguridad laboral percibida cuantitativa la media de la muestra es 10,97 ( $\mathrm{DE}=4,61)$. Un 25\% de los participantes presenta un alto nivel de preocupación frente a la posibilidad de perder su fuente laboral (ver Tabla 1). La puntuación media es mayor para los empleados que tienen un nivel de escolaridad bajo

Tabla 1.

Puntuaciones en las escalas de inseguridad laboral percibida por niveles, en frecuencias y porcentajes.

\begin{tabular}{|c|c|c|}
\hline Inseguridad Laboral Percibida & N & $\%$ \\
\hline \multicolumn{3}{|l|}{ Cuantitativa } \\
\hline Baja & 21 & 19 \\
\hline Mediana & 63 & 56 \\
\hline Alta & 28 & 25 \\
\hline Total & 112 & 100 \\
\hline \multicolumn{3}{|l|}{ Cualitativa } \\
\hline Ваја & 34 & 30 \\
\hline Mediana & 45 & 40 \\
\hline Alta & 33 & 30 \\
\hline Total & 112 & 100 \\
\hline
\end{tabular}

Tabla 2.

Puntuaciones en la escala de malestar psicológico por niveles, en frecuencias y porcentajes.

\begin{tabular}{|c|c|c|}
\hline Malestar Psicológico & $N$ & $\%$ \\
\hline Bajo & 27 & 24 \\
\hline Mediano & 57 & 51 \\
\hline Alto & 28 & 25 \\
\hline Total & 112 & 100 \\
\hline
\end{tabular}

que para quienes poseen un nivel alto $(\mathrm{M}=3,11 \mathrm{y} \mathrm{M}=2,44)$, siendo esta diferencia significativa $(F=3,193, p=0,045)$. En cambio, para las variables sexo, edad, situación de pareja y tipo de contrato no hay diferencias significativas.

En la escala de inseguridad laboral percibida cualitativa la media del grupo es 10,36 ( $\mathrm{DE}=3,19)$. Un 30\% de los participantes manifiesta un alto nivel de preocupación ante la posibilidad de perder alguna característica valorada de su trabajo (ver Tabla 1). En la variable sexo se observa una diferencia significativa $(t=-2,275$, $\mathrm{p}=0,025)$, siendo mayor la media de las mujeres que la de los hombres $(M=3,67$ y $M=3,22)$. Las diferencias observadas para las demás variables no son significativas.

En la escala de malestar psicológico la media en la puntuación total del grupo estudiado es 64,9 (DE $=19,7)$. Un 76\% de los participantes están ubicados en los niveles de mediano y alto malestar psicológico (ver Tabla 2). La media de las mujeres es mayor que la de los hombres $(\mathrm{M}=2,30 \mathrm{y} \mathrm{M}=1,86)$, y esa diferencia es significativa ( $t=-3,931, p=0,000)$. También en la variable edad hay diferencias significativas $(F=2,352, p=0,036)$, siendo la media del grupo de 53 a 59 años $(M=2,92)$ más alta que la de los grupos de 39 a $45(\mathrm{M}=1,84)$ y de 60 y más años ( $\mathrm{M}$ $=1,52$ ). Para las demás variables en estudio no existen diferencias significativas en el malestar psicológico.

\section{Resultados para el modelo teórico de Hellgren y Sverke ${ }^{24}$}

Este modelo propone que la inseguridad laboral percibida cuantitativa predice el malestar psicológico, y que los antecedentes sociodemográficos (sexo, edad, situación de pareja y escolaridad) y el tipo de contrato predicen la inseguridad laboral percibida cuantitativa y el malestar psicológico. En la Tabla 3 pueden observarse las medias y covarianzas entre las variables.

Los resultados con el índice Chi-cuadrado señalan diferencias significativas entre el modelo estimado y los datos $\left(\_=133.814\right.$, $\mathrm{p}=0,000$ ). Los otros índices de ajuste global muestran de forma general que el modelo tiene un ajuste pobre, puesto que los

Tabla 3.

Matriz de covarianzas con medias y desviaciones estándares de variables observables.

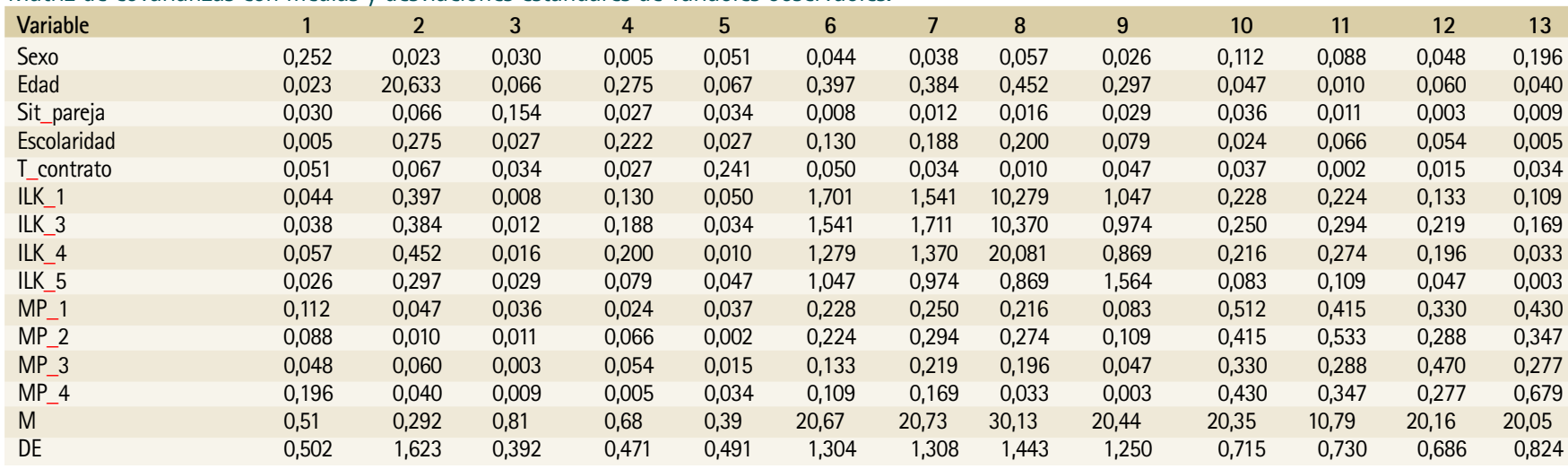

$\mathrm{n}=112 \quad$ ILK $=$ Inseguridad Laboral Percibida Cuantitativa $\quad \mathrm{MP}=$ Malestar Psicológico. 
Tabla 4.

Resumen de los estadísticos de ajuste del modelo propuesto por Hellgren y Sverke (2003).

$\begin{array}{lccccccc}\text { Modelo } & 2 & \text { gl } & p & \text { 2/gl } & \text { RMSEA } & \text { GFI } & \text { CFI } \\ \text { Modelo estructural } & 133,814 & 59 & 0,000 & 2,268 & 0,301 & 0,449 & 0,806 \\ \text { Modelo saturado } & 0,000 & 0 & & & & 1,000 & 1,000 \\ \text { Modelo independiente } & 462,893 & 78 & 0,000 & 5,935 & 0,594 & 0,130 & 0,000 \\ \mathrm{n}=112 & & & & & & & \end{array}$

valores de RMSEA, GFI y CFI están fuera de los límites de aceptación establecidos. Del conjunto de índices de ajuste revisados sólo la Razón entre Chi-cuadrado y los grados de libertad presenta un valor aceptable $(\angle 2 / g l=2.268)$. Detalles de estos índices se ven en la Tabla 4.

En suma, los resultados de la evaluación global del modelo estructural indican que este modelo teórico no tiene un buen ajuste con los datos observados y, por tanto, la configuración de variables que da forma al modelo no predice el malestar psicológico en los trabajadores suministrados chilenos. Las hipótesis derivadas de este modelo se rechazan.

La Figura 1 muestra el modelo estructural con sus correspondientes estimaciones estandarizadas y las varianzas explicadas para cada variable endógena.

\section{Resultados en el modelo teórico modificado por Sverke et al. ${ }^{3}$}

El modelo modificado propone que la inseguridad laboral percibida cualitativa predice el malestar psicológico, y que los antecedentes sociodemográficos (sexo, edad, situación de pareja y escolaridad) y el tipo de contrato predicen la inseguridad laboral percibida cualitativa y el malestar psicológico. La Tabla 5 muestra las medias y covarianzas entre las variables.

Los resultados con el índice Chi-cuadrado señalan diferencias significativas entre el modelo estimado y los datos $\_2=116.957$, $\mathrm{p}=0,000)$. Los otros índices de ajuste global muestran en forma general un ajuste pobre del modelo porque los valores de RMSEA, GFI y CFI se encuentran fuera de los límites de aceptación establecidos. En suma, de todos los índices de ajuste revisados sólo la Razón entre Chi-cuadrado y los grados de libertad presenta un valor aceptable $\left(\_2 / \mathrm{gl}=2.437\right)$. La Tabla 6 muestra en detalle los índices para analizar la validez del modelo.

Los resultados indican que tampoco este modelo teórico es apli-
Figura 1.

Modelo estructural de la relación entre inseguridad laboral percibida cuantitativa y malestar psicológico, y sus variables predictoras. Estimaciones estandarizadas para las proporciones de varianza explicada.

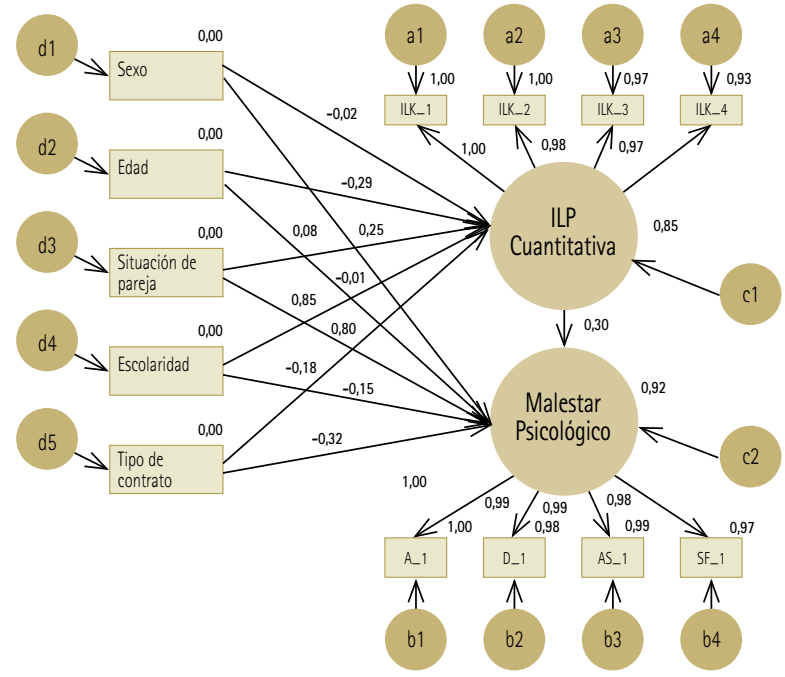

cable a los trabajadores suministrados chilenos, puesto que la evaluación global del modelo estructural indica que no tiene un buen ajuste con los datos observados, implicando que la configuración de variables que lo constituye no predice el malestar psicológico. Lo anterior lleva a rechazar las hipótesis derivadas del modelo teórico modificado.

La Figura 2 muestra el modelo estructural con sus correspondientes estimaciones estandarizadas y las varianzas explicadas para cada variable endógena.

\section{Tabla 6.}

Resumen de los estadisticos de ajuste del modelo modificado por Sverke et al. (2006).

$\begin{array}{lccccccc}\text { Modelo } & 2 & \mathrm{gl} & p & 2 / \mathrm{gl} & \mathrm{RMSEA} & \mathrm{GFI} & \mathrm{CFI} \\ \text { Modelo estructural } & 116,957 & 48 & 0,000 & 2,437 & 0,332 & 0,455 & 0,778 \\ \text { Modelo saturado } & 0,000 & 0 & & & & 1,000 & 1,000 \\ \text { Modelo independiente } & 376,629 & 66 & 0,000 & 5,706 & 0,602 & 0,124 & 0,000 \\ \mathrm{n}=112 & & & & & & & \end{array}$

Tabla 5.

Matriz de covarianzas con medias y desviaciones estándares de variables observables.

$\begin{array}{lcccccccccccc}\text { Variable } & 1 & 2 & 3 & 4 & 5 & 6 & 7 & 8 & 9 & 10 & 11 & 12 \\ \text { Sexo } & 0,252 & 0,023 & 0,030 & 0,005 & 0,051 & 0,069 & 0,132 & 0,138 & 0,112 & 0,088 & 0,048 & 0,196 \\ \text { Edad } & 0,023 & 20,633 & 0,066 & 0,275 & 0,067 & 0,329 & 0,120 & 0,029 & 0,047 & 0,010 & 0,060 & 0,040 \\ \text { Sit_pareja } & 0,030 & 0,066 & 0,154 & 0,027 & 0,034 & 0,054 & 0,055 & 0,030 & 0,036 & 0,011 & 0,003 & 0,009 \\ \text { Escolaridad } & 0,005 & 0,275 & 0,027 & 0,222 & 0,027 & 0,030 & 0,146 & 0,010 & 0,024 & 0,066 & 0,054 & 0,005 \\ \text { T_contrato } & 0,051 & 0,067 & 0,034 & 0,027 & 0,241 & 0,049 & 0,041 & 0,075 & 0,037 & 0,002 & 0,015 & 0,034 \\ \text { ILC_1 } & 0,069 & 0,329 & 0,054 & 0,030 & 0,049 & 1,539 & 0,982 & 0,763 & 0,188 & 0,172 & 0,081 & 0,154 \\ \text { ILC_2 } & 0,132 & 0,120 & 0,055 & 0,146 & 0,041 & 0,982 & 1,624 & 0,971 & 0,306 & 0,239 & 0,203 & 0,220 \\ \text { ILC44 } & 0,138 & 0,029 & 0,030 & 0,010 & 0,075 & 0,763 & 0,971 & 1,547 & 0,332 & 0,287 & 0,219 & 0,322 \\ \text { MP_1 } & 0,112 & 0,047 & 0,036 & 0,024 & 0,037 & 0,188 & 0,306 & 0,332 & 0,512 & 0,415 & 0,330 & 0,430 \\ \text { MP_2 } & 0,088 & 0,010 & 0,011 & 0,066 & 0,002 & 0,172 & 0,239 & 0,287 & 0,415 & 0,533 & 0,288 & 0,347 \\ \text { MP_3 } & 0,048 & 0,060 & 0,003 & 0,054 & 0,015 & 0,081 & 0,203 & 0,219 & 0,330 & 0,288 & 0,470 & 0,277 \\ \text { MP_4 } & 0,196 & 0,040 & 0,009 & 0,005 & 0,034 & 0,154 & 0,220 & 0,322 & 0,430 & 0,347 & 0,277 & 0,679 \\ \text { M } & 0,51 & 0,292 & 0,81 & 0,68 & 0,39 & 3,290 & 3,380 & 30,70 & 20,35 & 10,79 & 20,16 & 20,05 \\ \text { DE } & 0,502 & 1,623 & 0,392 & 0,471 & 0,491 & 1,241 & 1,274 & 1,244 & 0,715 & 0,730 & 0,686 & 0,824\end{array}$

$\mathrm{n}=112 \quad$ ILK $=$ Inseguridad Laboral Percibida Cuantitativa $\quad \mathrm{MP}=$ Malestar Psicológico. 


\section{Figura 2.}

Modelo estructural de la relación entre inseguridad laboral percibida cualitativa y malestar psicológico, y sus variables predictoras. Estimaciones estandarizadas para las proporciones de varianza explicada.

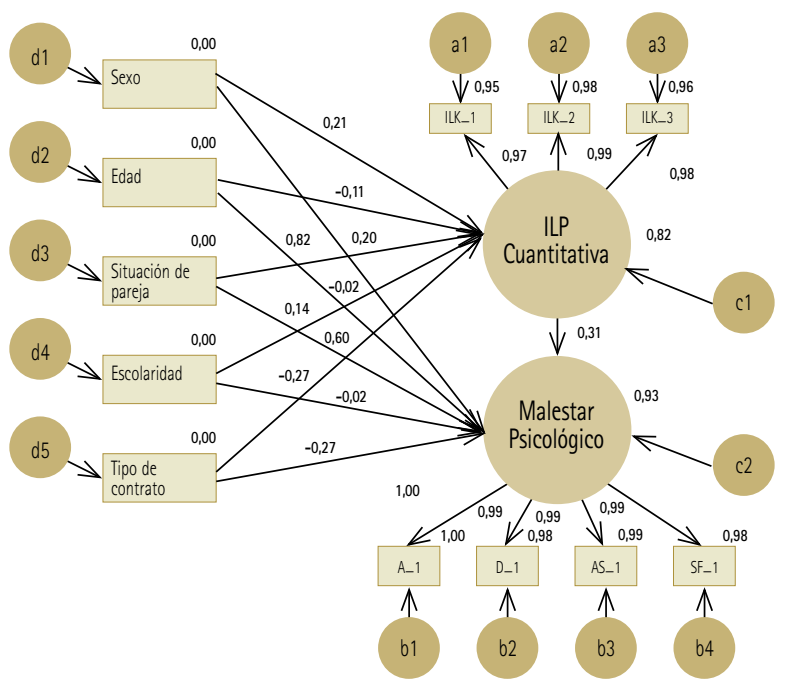

\section{DISCUSIÓN}

Esta investigación tuvo como objetivo probar dos modelos teóricos desarrollados en Suecia sobre la relación entre inseguridad laboral percibida y malestar psicológico, y sus variables predictoras, en trabajadores suministrados chilenos. Los resultados muestran que el modelo teórico referido a la relación entre inseguridad laboral percibida cuantitativa y malestar psicológico, propuesto por Hellgren y Sverke ${ }^{24}$, no se confirma con trabajadores suministrados chilenos. Tampoco se confirma el modelo teórico modificado por Sverke et $\mathrm{al}^{3}$ en que la variable inseguridad laboral percibida cuantitativa es reemplazada por la variable inseguridad laboral percibida cualitativa. Los resultados obtenidos señalan la necesidad de formular otros modelos teóricos que expliquen esta relación en trabajadores suministrados chilenos.

Estos resultados podrían deberse a las diferencias en las situaciones de trabajo y las formas de flexibilidad laboral de los participantes en que fueron probados ambos modelos. Hellgren y Sverke formularon el modelo original a partir de trabajadores del retail que atravesaban un proceso de reducción de personal (una forma de flexibilidad cuantitativa del trabajo), mientras en esta investigación los modelos fueron probados en trabajadores suministrados, lo que supone una transformación cualitativa del sistema productivo de la organización y la relación salarial mediante una estrategia de externalización. ${ }^{10}$ Algunas variables asociadas con las características específicas de la situación de trabajo suministrado, como la relación laboral triangular o las asignaciones a distintas empresas usuarias ${ }^{11}$, podrían afectar la relación entre inseguridad laboral y malestar psicológico.

También son relevantes las diferencias en las condiciones estructurantes de los contextos laborales específicos, donde variables como el marco legislativo del trabajo suministrado y las características del mercado laboral juegan un rol determinante. Echeverría et $\mathrm{al}^{36}$ encontraron en Chile que, además del suministro de personal temporal, el suministro de personal permanente está utilizándose para ocupar puestos transitorios de trabajo. La legislación chilena no garantiza que el trabajo suministrado sea efectivamente de "misiones" temporales, y que no se convierta en una forma habitual de contratación, prohibido internacionalmente porque, generalmente, encubre las verdaderas relaciones de trabajo, incorporando al suministrador como fachada y precarizando el empleo. Por otra parte, el mercado laboral presenta problemas graves en la calidad del empleo, con marcada tendencia a la informalidad, inestabilidad y desprotección, implicando para los trabajadores una "inserción laboral endeble"; además, el "Índice de Desempleo Integral", que incluye a los desempleados y subempleados, refleja que el aumento efectivo del subempleo en el país no estaría declarado en las cifras oficiales de desempleo., ${ }^{5,37}$ En cambio, el mercado laboral sueco presenta más oportunidades de acceder a un empleo más protegido y estable, en particular para las mujeres. ${ }^{38,39}$

Los resultados obtenidos deben, sin embargo, considerarse a la luz de las limitaciones del presente estudio. Primero, faltan items que aludan a las relaciones sociales establecidas en el trabajo ${ }^{3}$ y a las condiciones específicas del trabajo suministrado en Chile. Segundo, aunque el tamaño de la muestra cumple con el mínimo recomendado de ser superior a $100^{33-35}$, hay consenso que sobre 200 mejoran las garantías de validez en el análisis SEM. Finalmente, la selección muestral intencionada no permite, por cierto, la representatividad ni la generalización.

En el estudio de la relación entre inseguridad laboral percibida y malestar psicológico en trabajadores de EST debieran incorporarse otras variables en el nivel individual que podrian afectar la relación, configurando escenarios laborales "percibidos" diferentes para los trabajadores, como las motivaciones y la voluntariedad para tomar un empleo suministrado. ${ }^{9}$ Asimismo, examinar variables que podrían ser predictoras o amortiguar los efectos negativos sobre el malestar psicológico tales como empleabilidad y apoyo organizacional ${ }^{21}$, control percibido en el trabajo ${ }^{40}$, percepción de dependencia al trabajo ${ }^{41}$, responsabilidad familiar ${ }^{3} \mathrm{y}$ estrategias de afrontamiento del estrés. ${ }^{16}$ Por último, estudios longitudinales permitirían ver la evolución de los efectos de la inseguridad laboral sobre el malestar psicológico de trabajadores suministrados en su trayectoria laboral.

Esta investigación se realizó en el Departamento de Psicología de la Facultad de Ciencias Sociales de la Universidad de Concepción. 
1. Goldberg DP, Williams P. A user's guide to the general health questionnaire. Windsor: NFER-Nelson; 1988.

2. Boya $F$, Demiral $Y$, Ergor A, Akvardar Y, De Witte H. Effects of perceived job insecurity on perceived anxiety and depression in nurses. Ind Health. 2008;46: 613-619.

3. Sverke M, Hellgren J, Näswall K. Job insecurity: A literature review. Stockholm: The National Institute for Working Life; 2006.

4. Chile. Dirección del Trabajo. Encuesta laboral 2008[en línea]. Santiago: Dirección del Trabajo; 2008 [citado 7 Ago 2011]. Disponible en: http://www. dt.gob.cl/documentacion/1612/articles-95958_archivo_fuente.pdf.

5. Fundación Sol. Minuta de empleo N³9, Agosto-Octubre 2013[en línea]. Santiago: Fundación Sol; 2013 [citado 2 nov 2013]. Disponible en: http:// www.fundacionsol.cl/wp-content/uploads/2011/02/Minuta-AgostoOctubre-2010.pdf.

6. Chile. Dirección del Trabajo. Encuesta laboral 2011 [en línea]. Santiago: Dirección del Trabajo; 2011. [citado 9 jun 2013]. Disponible en: http://www. dt.gob.cl/documentacion/1612/articles-101347_recurso_1.pdf.

7. Galais N, Moser K. Organizational commitment and the well-being of temporary agency workers: A longitudinal study. Hum Relat. 2009;62(4):589-620.

8. Black N. Temporary agency workers: What are their rights and what are yours? Eng Manage. 2006;16(1):32-33.

9. Martínez G, De Cuyper N, De Witte H. Review of temporary employment in literature: Perspectives for research and development in Latin America. Psykhe. 2010;19(1):61-73.

10. Soto A, Espinoza G, Gómez J. Los aspectos subjetivos de la flexibilidad laboral. En: Soto A, editor. Flexibilidad laboral y subjetividades: Hacia una comprensión psicosocial del empleo contemporáneo. Santiago: LOM; 2008. p. 1-37.

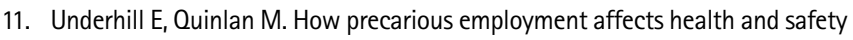
at work: The case of temporary agency workers. Relat Ind. 2011;66(3):397-421.

12. Sverke $M$, Hellgren J, Näswall $K$. No security: A meta-analysis and review of job insecurity and its consequences. J Occup Health Psychol. 2002; 7:242-264.

13. Greenhalgh L, Rosenblatt Z. Job insecurity: Toward conceptual clarity. Acad Manage Rev. 1984;3:438-448.

14. Kinnunen $U$, Feldt T, Mauno S. Job insecurity and self-esteem: Evidence from cross-lagged relations in a 1-year longitudinal sample. Pers Individ Differ. 2003;35: 617-632.

15. Hellgren J, Sverke M, Isaksson K. A two-dimensional approach to job insecurity: Consequences for employee attitudes and well-being. Eur J Work Organ Psychol. 1999;8:179-195.

16. Cheng T. Revisiting the buffers of job insecurity: Investigating new buffering factors between perceived job insecurity and employee outcomes. Finland: University of Jyväskylä; 2013. (Jyväskylä Studies in Education, Psychology and Social Research; 90).

17. Burgard S, Brand J, House J. Job insecurity and health in the United States. Michigan: Population Studies Center Research; 2006.

18. Clark L. Moderators of the effects of perceived job insecurity: A comparison of temporary and permanent employees (Manuscrito no publicado). Brisbane: Queensland University of Technology; 2005.

19. Strazdins L, D'Souza R, Lim L, Broom D, Rodgers B. Job strain, job insecurity and health: Rethinking the relationship. J Occup Health Psychol. 2004;9:296-305.

20. Kriese P. Job insecurity and self-efficacy in a chemical industry. Programme in Industrial Psychology. (Manuscrito no publicado). Vanderbijlpark: North-West University; 2008.

21. Silla I, De Cuyper N, Gracia F, Peiró J, De Witte H. Job insecurity and wellbeing: Moderation by employability. J Happiness Stud. 2009;10:739-775.
22. Rocha $\mathrm{C}$, Hause J, McCarter A. The effects of prolonged job insecurity on the psychological well-being of workers. J Sociol Soc Welfare. 2006;33(3):9-2.

23. Lewchuk W, Clarke M, De Wolff A. Working without commitments: Precarious employment and health. Work Employ Soc. 2008;22:387-406.

24. Hellgren J, Sverke M. Does job insecurity lead to impair well-being or vice versa?: Estimation of cross-lagged effects using latent variable modelling. J Organ Behav. 2003;24:215-236.

25. Maurier W, Northcott $\mathrm{H}$. Job uncertainty and health status for nurses during restructuring of health care in Alberta. West J Nurs Res. 2000; 22:623-641.

26. Viljoen E. Job insecurity, burnout, job engagement and psychological wellbeing of workers at a government organization. [Manuscrito no publicado], Gauteng: North-West University-School of Behavioural Sciences; 2004.

27. Chambel $M$, Fontinha R. Contingencies of contingent employment: Psychological contract, job insecurity and employability of contracted workers. Revista de Psicología del Trabajo y de las Organizaciones. 2009;25:207-217.

28. Cheng $G$, Chan D. Who suffers more from job insecurity?: A meta-analytic review. Appl Psychol. 2008;57:272-303.

29. Ashford $S$, Lee $C$, Bobko P. Content, causes and consequences of job insecurity: A theory-based measure and substantive test. Acad. Manage J. 1989; 32(4):803-829.

30. De Witte $\mathrm{H}$. Work ethic and job insecurity: measurement and consequences for well-being, satisfaction and performance. In: Bouwen $R$, De Witte $H_{\text {, }}$ Taillieu T, eds. Leuven: Van groep naar gemeenschap; 2000. p.325-350

31. Isackson K, Hellgren J, Pettersson P. Structural transformation in Swedish retailtrade: Follow-up of a reorganization and layoff in KF/KDAB. Stockholm: Stockholm University; 1998. Reports from the Department of Psychology; 97.

32. Avendaño $C$, Román J. Efectos de los roles múltiples en el bienestar psicológico en enfermeras chilenas. Psykhe. 2002;11(2):27-42.

33. Weston R, Gore PA. A brief guide to structural equation modeling. Couns Psychol. 2006;34:719-751.

34. Ruiz MA, Pardo A, San Martín R. Modelos de ecuaciones estructurales. Pap Psicol. 2010;31(1):34-45.

35. Kline RB. Principles and practice of structural equation modeling. 3a ed. New York: The Guilford Press; 2011

36. Echeverria M, Solis V, Ugarte JL, Uribe-Echeverría V. El otro trabajo: El suministro de personas en las empresas. Santiago: Dirección del Trabajo; 2001.

37. Fundación Sol. Precariedad laboral y modelo productivo en Chile [en línea]. Santiago: Fundación Sol; 2011. [citado 2 nov 2013]. Disponible en: http:// www.fundacionsol.cl/wp-content/uploads/2011/12/Ideas-1-Tendencias-delTrabajo2.pdf.

38. Organización para la Cooperación y el Desarrollo Económicos Índice para una vida mejor, Chile [en línea]. Paris: OCDE; 2013a. [citado 3 nov 2013]. Disponible en: http://www.oecdbetterlifeindex.org/es/countries/chile-es/.

39. Organización para la Cooperación y el Desarrollo Económicos Índice para una vida mejor, Suecia [en línea]. Paris: OCDE; (2013b). [citado 3 nov 2013]. Disponible en: http://www.oecdbetterlifeindex.org/es/countries/sweden-es/.

40. Schreurs B, Van Emmerik $H$, Notelaers G, De Witte $H$. Job insecurity and employee health: The buffering potencial of job control and job self-efficacy. Work Stress. 2010;24(1):56-72.

41. Richter A, Näswall K, Bernhard-Oettel C, Sverke M. Job insecurity and wellbeing: The moderating role of job dependence. Eur J Work Organ Psychol [on line] 2014 [citado 3 nov 2013]. Disponible en: http://dx.doi.org/10.1080/1359 432X.2013.805881.

Referencia complementaria:

-Echeverría M. Los riesgos laborales de la subcontratación. Santiago: Dirección del Trabajo; 2006. 